\title{
STATISTICAL ESTIMATES OF THE PARAMETERS OF
} STABLE LAWS

\section{M. ZOLOTAREV}

Steklov Mathematical Institute, Moscow, U.S.S.R.

\section{Introduction}

The set $\mathfrak{U}$ of all possible limit laws of linearly normalized sums of an infinitely increasing number of independent and identically distributed random variables is called the class of stable laws. Further, the letters $G, f$ will be used to denote distribution functions and characteristic functions of the stable laws, respectively. Letter $X$ will denote a random variable having a stable probability law.

It turns out that the most convenient way of describing all distributions from the class $\mathfrak{U}$ is to use characteristic functions of those distributions.

A form proposed by A. Ya. Khintchine (see for ex. [4]) is the most common form to record the function $f$. This form will be called form (A):

where

$$
\log f(t)=i t \gamma-\lambda|t|^{\alpha} \omega_{A}(t, \alpha, \beta),
$$

$$
\omega_{A}(t, \alpha, \beta)= \begin{cases}1-i \beta \tan \left(\frac{\pi}{2} \alpha\right) \operatorname{sign} t & \text { if } \quad \alpha \neq 1, \\ 1+i \beta \frac{2}{\pi} \log |t| \operatorname{sign} t & \text { if } \quad \alpha=1 .\end{cases}
$$

The parameters which are present in the above formula change within the following bounds:

$$
0<\alpha \leqslant 2, \quad-1 \leqslant \beta \leqslant 1, \quad-\infty<\gamma<\infty, \quad \lambda \geqslant 0 .
$$

Hence, the set $\mathfrak{U}$ is a four-parameters family of the probability laws. Class $\mathfrak{U}$ contains such well-known probability laws as the normal law $(\alpha=2)$, the Cauchy law $(\alpha=1$, $\beta=0)$ and the Lévy law $(\alpha=1 / 2, \beta=1)$.

The above-mentioned probability laws together with a law similar to the Lévy law $(\alpha=1 / 2, \beta=-1)$ are the only laws whose densities $g$ can be expressed with the help of the elementary functions (including of course those variants of stable laws which appear when we change the values of the nonfixed parameters $\gamma, \lambda$ ). 
Stable probability laws appear in applications to astronomy, physics and technology. So the problem of constructing statistical estimates of the parameters describing them is becoming more and more the problem of the day. At present there exist several papers devoted to the problem of estimating the parameters of stable laws. Some of them have a general character and make use either of the method of maximum likelihood [7] or of the method of moments [6]. There do exist also papers oriented toward applications; namely, they deal with the construction of specific calculating algorithms for the estimation of the parameters $\alpha$ and $\lambda$ ([2], [3], [6], [12], [13]).

The main difficulty in the application of the results of the well-developed theory of estimation of the parameters is connected with the fact that the explicit formulae for the densities of stable laws are known only for the few cases mentioned above.

The construction of the stable law parameter estimates stems from the method of moments, well-known in statistics, and is based on the sequence of analytical properties of the stable distribution described in the present author's paper [8]. The solution of this problem is presented below.

\section{Some properties of the distributions from the class $\mathfrak{U}$}

We shall start presentation of the properties of the stable distributions from writing the function $f$ in another form. In many cases this new form turns out to be more convenient from the analytical point of view.

This form is believed to be obtained by the author for the first time in [8] and, in a little bit changed form, in [9]. It will be called further form (B):

where

$$
\log f(t)=i t \gamma-\lambda|t|^{\alpha} \omega_{B}(t, \alpha, \beta),
$$

$$
\omega_{B}(t, \alpha, \beta)= \begin{cases}\exp \left\{-i \frac{1}{2} \pi \beta K(\alpha) \operatorname{sign} t\right\} & \text { if } \quad \alpha \neq 1 \\ 1+i \beta \log |t| \operatorname{sign} t & \text { if } \quad \alpha=1\end{cases}
$$

We denote here $K(\alpha)=1-|1-\alpha|=\min (\alpha, 2-\alpha)$ and the parameters $\alpha, \beta, \gamma, \lambda$ can be changed within the same intervals as in form (A).

Parameters appearing in forms (A) and (B) are related by the following formulae (indices $\mathrm{A}$ and $\mathrm{B}$ of the parameters denote their membership in one or another form):

If $\alpha \neq 1$, then

$$
\alpha_{A}=\alpha_{B}=\alpha ; \quad \gamma_{A}=\gamma_{B}=\gamma
$$

$$
\begin{gathered}
\beta_{\mathrm{A}}=\cot \left(\frac{1}{2} \pi \alpha\right) \tan \left[\frac{1}{2} \pi K(\alpha) \beta_{\mathrm{B}}\right], \\
\lambda_{\mathrm{A}}=\lambda_{B} \cos \left[\frac{1}{2} \pi K(\alpha) \beta_{\mathrm{B}}\right] .
\end{gathered}
$$

If $\alpha=1$ then

$$
\beta_{\mathrm{A}}=\beta_{\mathrm{B}}, \quad \lambda_{\mathrm{A}}=\lambda_{\mathrm{B}}
$$

The four simple properties of stable laws given below will be formulated in terms of the random variables $X(\alpha, \beta, \gamma, \lambda)$, as it turns out to be more convenient (properties will be more readable and illustrative). Parameters in brackets following the symbol of the random variable are related to the distributions of those random variables. The equality between random variables $(\stackrel{d}{=})$ is understood as the equality between the corresponding distributions. Further random variables appearing on one side of the d-equality are assumed to be independent.

Property (2.1). For any possible values of the parameters, we have

$$
X(\alpha, \beta, \gamma, \lambda) \stackrel{\mathrm{d}}{=} a X(\alpha, \beta, 0,1)+b .
$$

We have here (irrespective of the form to which those parameters are related): $a=\lambda^{1 / \alpha}$ and $b=\gamma$ if $\alpha \neq 1$ and $b=\gamma+(2 / \pi) \beta \lambda \log \lambda$ if $\alpha=1$.

The above property shows that parameters $\gamma$ and $\lambda$ are connected with the choice of scale and the zero point within the set of values of random variable $X$.

PROPERTY (2.2). For any admissible values of the parameters (irrespective of the form) we have

$$
X(\alpha,-\beta,-\gamma, \lambda) \stackrel{d}{=}-X(\alpha, \beta, \gamma, \lambda)
$$

PROPERTY (2.3). For any two admissible systems of the parameters $\left(\alpha, \beta^{\prime}, \gamma^{\prime}, \lambda^{\prime}\right)$, $\left(\alpha, \beta^{\prime \prime}, \gamma^{\prime \prime}, \lambda^{\prime \prime}\right)$ and any real numbers $c^{\prime}, c^{\prime \prime}, h$ one can find a system of the parameters $(\alpha, \beta, \gamma, \lambda)$ such that

$$
X(\alpha, \beta, \gamma, \lambda) \stackrel{\mathrm{d}}{=} c^{\prime} X\left(\alpha, \beta^{\prime}, \gamma^{\prime}, \lambda^{\prime}\right)+c^{\prime \prime} X\left(\alpha, \beta^{\prime \prime}, \gamma^{\prime \prime}, \lambda^{\prime \prime}\right)+h .
$$

The relation between the system of parameters $\alpha, \beta, \gamma, \lambda$ and the random variables describing them turns out to be simplest in the case where form (A) is used:

$$
\begin{aligned}
\lambda & =\lambda^{\prime}\left|c^{\prime}\right|^{\alpha}+\lambda^{\prime \prime}\left|c^{\prime \prime}\right|^{\alpha}, \\
\beta \lambda & =\beta^{\prime} \lambda^{\prime}\left|c^{\prime}\right|^{\alpha} \operatorname{sign} c^{\prime}+\beta^{\prime \prime} \lambda^{\prime \prime}\left|c^{\prime \prime}\right|^{\alpha} \operatorname{sign} c^{\prime \prime}, \\
\gamma & =c^{\prime} \gamma^{\prime}+c^{\prime \prime} \gamma^{\prime \prime}+h+h_{0},
\end{aligned}
$$

where $h_{0}=0$ if $\alpha \neq 1$ and

$$
h_{0}=-\frac{2}{\pi}\left(\beta^{\prime} \lambda^{\prime} c^{\prime} \log \left|c^{\prime}\right|+\beta^{\prime \prime} c^{\prime \prime} \lambda^{\prime \prime} \log \left|c^{\prime \prime}\right|\right) \quad \text { if } \quad \alpha=1 .
$$

We shall mention three particular cases of the last property, assuming that all the parameters are related to the form (A)

(2.3a): For any admissible values of the parameters,

$$
X(\alpha, \beta, \gamma, \lambda)-X(\alpha, \beta, \gamma, \lambda) \stackrel{d}{=} X(\alpha, 0,0,2 \lambda) .
$$

(2.3b): For any admissible values of the parameters, where

$$
\begin{gathered}
X(\alpha, \beta, \gamma, \lambda)-\frac{1}{2} X(\alpha, \beta, \gamma, \lambda)-\frac{1}{2} X(\alpha, \beta, \gamma, \lambda) \stackrel{\mathrm{d}}{=} X\left(\alpha, \beta^{*}, \gamma^{*}, \lambda^{*}\right), \\
\lambda^{*}=\left(1+2^{1-\alpha}\right) \lambda, \quad \beta^{*} \lambda^{*}=\left(1-2^{1-\alpha}\right) \beta \lambda,
\end{gathered}
$$

(obviously we have $\left|\beta^{*}\right|<1$ ); $\gamma^{*}=0$ if $\alpha \neq 1$ and $\gamma^{*}=-\beta \lambda \frac{2}{\pi} \log 2$ if $\alpha=1$. 
(2.3c): For any admissible choice of the parameters,

where

$$
X(\alpha, \beta, \gamma, \lambda) \stackrel{\mathrm{d}}{=} c_{1} X(\alpha, 1,0,1)-c_{2} X(\alpha, 1,0,1)+l
$$

$$
\begin{aligned}
& c_{1}=\left(\lambda \frac{1+\beta}{2}\right)^{1 / \alpha}, \quad c_{2}=\left(\lambda \frac{1-\beta}{2}\right)^{1 / \alpha}, \\
& l=\left\{\begin{array}{l}
\gamma \quad \text { if } \quad \alpha \neq 1, \\
\frac{2}{\pi}\left(c_{1} \log c_{1}-c_{2} \log c_{2}\right)+\gamma \quad \text { if } \quad \alpha=1 .
\end{array}\right.
\end{aligned}
$$

The properties (2.1)-(2.3) can be found in paper [8]. However, those relations between stable distributions can easily be checked by the reader himself. This can be done by equalizing the left and right-hand sides of the above quoted formulae.

As can easily be seen from the form of function $f$, stable laws are continuous (in the sense of weak and even strong convergence of the distributions) with respect to the parameters $(\beta, \gamma, \lambda)$ within the region of all their admissible values. They are not continuous, however, with respect to the parameter $\alpha$ in each neighbourhood of any point $(\alpha=1, \beta \neq 0)$. As $\alpha \rightarrow 1$ and the values of all other parameters are fixed $(\beta \neq 0)$, the whole mass of the probability distribution diverges to infinity if the parameters are related to form (A), and concentrates at one point if the parameters are related to form (B).

Continuity with respect to parameter $\alpha$ in the neighbourhood of the point $\alpha=1$, however, can be reached in the most convenient way by using form (A). It suffices to construct an additional shift of the distribution which would compensate the divergence of the probability mass to infinity.

PROPERTY (2.4). If two parameter systems $\left(\alpha^{\prime}, \beta^{\prime}, \gamma^{\prime}, \lambda^{\prime}\right)$ and $(1, \beta, \gamma, \lambda)$ related to form (A) change in such a way that

then

$$
\begin{gathered}
1 \neq \alpha^{\prime} \rightarrow 1, \quad \beta^{\prime} \rightarrow \beta, \quad \gamma^{\prime} \rightarrow \gamma, \quad \lambda^{\prime} \rightarrow \lambda, \\
X\left(\alpha^{\prime}, \beta^{\prime}, \gamma^{\prime}, \lambda^{\prime}\right)-\lambda^{\prime} \beta^{\prime} \tan \frac{1}{2} \pi \alpha^{\prime} \rightarrow X(1, \beta, \gamma, \lambda),
\end{gathered}
$$

where the convergence of random variables is understood in the sense of the convergence of the related distributions. This convergence is either in the Lévy metric in the general case or in the uniform metric of $\lambda>0$.

The above limit property is presented in [8]. One can check it, however, without any difficulty by observing the convergence of the appropriate characteristic functions. The fact that for $\lambda>0$ the convergence can be understood in the strong sense or even as the convergence of the variances results from the following property of stable laws. Namely, it turns out that stable laws are absolutely continuous with any number of everywhere continuous derivatives.

\section{Stable laws of the subclass $\mathfrak{W}$}

Subclass $\mathfrak{B}$ of the class $\mathfrak{U}$ is defined by the following additional condition:

$$
G(x, \alpha, \beta, \gamma, \lambda) \in \mathfrak{W} \text {, }
$$

if $\gamma=0$ in case where $\alpha \neq 1$ or $\beta=0$ if $\alpha=1$. The set $\mathfrak{B}$ is of course a threeparameter family of stable laws. It turns out to be more convenient to describe this family by another system of parameters which do not appear in forms (A) and (B). This new system of parameters $(\nu, \theta, \tau)$ is related to the parameters $(\alpha, \beta$, $\gamma, \lambda)$ appearing in form (B) by the following relations:

$$
\begin{gathered}
\nu=\alpha^{-2}, \quad \theta=\left\{\begin{array}{l}
\frac{K(\alpha)}{\alpha} \beta, \quad \text { if } \quad \alpha \neq 1, \\
\frac{2}{\pi} \arctan (\gamma / \lambda) \quad \text { if } \quad \alpha=1,
\end{array}\right. \\
\tau=-\frac{1}{2 \alpha} \log \left(\lambda^{2}+\gamma^{2}\right)+C\left(\frac{1}{\alpha}-1\right),
\end{gathered}
$$

where $C=-\Gamma^{\prime}(1)=0.577 \ldots$ is the Euler constant.

Characteristic functions related to stable laws of the class $\mathfrak{B}$ can be written in the following way ((form $(C))$ :

$$
\log f(t)=-\exp \left\{\nu^{-1 / 2}\left[\log |t|+\tau-i \frac{1}{2} \pi \theta \operatorname{sign} t+C\left(1-\nu^{1 / 2}\right)\right]\right\},
$$

where parameters $\nu, \theta, \tau$ can be changed within the following bounds:

$$
\nu \geqslant \frac{1}{4}, \quad|\theta| \leqslant \min (1,2 \sqrt{\nu}-1), \quad-\infty<\tau<\infty .
$$

One can easily notice that all stable laws belonging to the set $\mathfrak{M}$ are continuous with respect to the parameters $(v, \theta, \tau)$ on the set of all their admissible values.

The above form of denoting the laws of class $\mathfrak{B}$ is not obviously superior to forms (A) and (B) except, of course, for the fact that in this form there are no discontinuity points. There is, however, one property of the laws from class $\mathfrak{B}$ which turns out to be very important in solving the problem of parameter estimation. This property is connected with so-called characteristic transformations of the real random variables, which play the same role with respect to the operation of multiplying independent random variables as characteristic functions play with respect to summation (see for it [10] and [11]).

According to its definition the characteristic transformation of the random variable $Y$ is a diagonal matrix

$$
W_{Y}(t)=\left[\begin{array}{cc}
w_{0} & 0 \\
0 & w_{1}
\end{array}\right], \quad w_{k}(t)=E|Y|^{i x}(\operatorname{sign} Y)^{k},
$$

where it is assumed that $0^{i t}=0$ for every $t$.

It turns out that for stable laws of class $\mathfrak{B}$ the characteristic transformation $W_{X}$ has a specially simple form.

Since it is known (see [4]) that for any admissible values of the parameters we have

$$
E|X(\alpha, \beta, \gamma, \lambda)|^{s}<\infty, \quad-1<\operatorname{Re} s<\alpha,
$$

we will use, instead of the characteristic transformations themselves, their analytical extensions,

$$
W_{X}(-i s), \quad-1<\operatorname{Re} s<\alpha,
$$


denoting

$$
u(s)=E|X| s, \quad v(s)=E|X|^{s} \operatorname{sign} X .
$$

Expressions describing functions $u$ and $v$ were given in [8]. If one transforms the formulae obtained there by using parameters $(\nu, \theta, \tau)$, then one gets the following theorem as a result of that operation.

THEOREM 1. Assume that random variable $X(\nu, \theta, \tau)$ has a distribution belonging to class $\mathfrak{W}$. Functions $u, v$ can then be expressed in the following form:

$$
\begin{aligned}
& u(s)=\exp \{\tau s+(1-\sqrt{\nu}) C s\} \frac{\cos \frac{1}{2} \pi \theta s}{\cos \frac{1}{2} \pi s} \frac{\Gamma(1-s \sqrt{\nu})}{\Gamma^{\prime}(1-s)}, \\
& v(s)=\exp \{\tau s+(1-\sqrt{\nu}) C s\} \frac{\sin \frac{1}{2} \pi \theta s}{\sin \frac{1}{2} \pi s} \frac{\Gamma(1-s \sqrt{\nu})}{\Gamma(1-s)} .
\end{aligned}
$$

The above theorem allows us to get a number of interesting corollaries concerning the construction of stable laws, formulae for moments of logarithmic order, and so on. Some of them will be presented below.

THEOREM 2. Let $X(v, \theta, \tau)$ be a random variable whose distribution belongs to the set $\mathfrak{M}$. Let $\theta_{*}$ be such constant that $|\theta| \leqslant\left|\theta_{*}\right| \leqslant \theta_{0}=\min (1,2 \sqrt{\nu}-1)$.

Then the following decomposition is true:

$$
X(\nu, \theta, \tau) \stackrel{d}{=} e^{\tau} X\left(\nu, \theta_{*}, 0\right) Y,
$$

where the random variables on the right-hand side are independent and .

$$
Y=\left|X_{*}\right| \operatorname{sign} X_{*}, \quad X_{*}=X\left(1, \theta / \theta_{*}, 0\right) \text {. }
$$

In particular, if $\nu \geqslant 1$ and $\theta_{*}=1$, then

$$
X(\nu, \theta, \tau) \stackrel{\mathrm{d}}{=} X(\nu, 1,0) X(1, \theta, 0) e^{\tau} .
$$
Write:

Let $X=X(\nu, \theta, \tau)$ be a random variable with distribution belonging to $\mathfrak{W}$.

\section{We then have}

$$
\begin{array}{ll}
U=\operatorname{sign} X, & \bar{U}=U-E U, \\
V=\log |X|, & \bar{V}=V-E V .
\end{array}
$$

$$
u(s)=E \exp (s V), \quad v(s)=E U \exp (s V) .
$$

Having really simple expressions describing those functions, we can also get simple formulae for the mixed moments:

$$
E U^{2 r} V^{k}=E V^{k}, \quad E U^{2 r+1} V^{k}=E U V^{k} ; \quad r, k=0,1, \ldots
$$

$\left(U^{2}=1\right.$ with probability 1$)$ by expanding functions $u(s)$ and $v(s)$ into power series with respect to $s$. In particular, taking into account only the first two summands of those expansions, we note that

$$
E U=\theta, \quad E V=\tau, \quad E U V=\theta \tau=E U \cdot E V .
$$

The above formulae permit us to get expressions for central mixed moments $E \bar{U}^{r} \bar{V}^{k}$. This can be done in the most convenient way with the help of the following moments:

$$
L_{k}^{o}=E \bar{V}^{k}, \quad L_{k}^{1}=E U \bar{V}^{k} / E U, \quad k=0,1, \ldots
$$

If $\theta=E U=0$ one can get an expression for $L_{k}^{1}$ by letting $\theta \rightarrow 0$ (such a limit always exists).

The calculation of those moments can be made with the help of the semiinvariants (for $L_{k}^{0}$ ) related to them or their analogues (for $L_{k}^{1}$ ). To obtain this we will expand the logarithms of the functions $u, v$ into power series with respect to $s$. This can easily be done by recalling the expansions of the functions $\log \Gamma(1-z)$, $\log \left(\frac{\sin z}{z}\right)$ and $\log \cos z$ into power series (see [5], 8.342, 1.518).

As a result we get after a few transformations the following expansions:

$$
\log u(s)=\tau s+\sum_{k=2}^{\infty}\left[a_{k}^{0}\left(1-\theta^{k}\right)+b_{k}^{0}\left(v^{k / 2}-1\right)\right] \frac{s^{k}}{k !},
$$

where

$$
a_{k}^{0}=\frac{\pi^{k}}{k}\left(2^{k}-1\right) B_{k}, \quad b_{k}^{0}=\Gamma(k) \zeta(k) ;
$$

$B_{k}$ denote here Bernoulli numbers and $\zeta(p)$ the Riemann dzeta-function. Note that if $k$ is even, then

$$
b_{k}^{0}=\Gamma(k) \zeta(k)=\frac{\pi^{k}}{k} 2^{k-1} B_{k} .
$$

In the case where $k$ is odd the quantity $\zeta(k)$ can be made arbitrarily near to 1 as $k$ increases

$$
\zeta(3)=1.202 \ldots, \quad \zeta(5)=1.037 \ldots
$$

Hence we can use the asymptotic estimation

$$
b_{k}^{0} \approx \Gamma(k) \text {. }
$$

Similarly, for positive values of $\theta$ (the case of negative values of $\theta$ can be reduced to the case of positive values due to the property $X(\nu,-\theta, \tau) \stackrel{\mathrm{d}}{=}-X(\nu, \theta, \tau)$, for the case $\theta=0$ we have $v(s) \equiv 0$ ), we get:

where

$$
\begin{gathered}
\log v(s)=\log \theta+\tau s+\sum_{k=2}^{\infty}\left[a_{k}^{1}\left(1-\theta^{k}\right)+b_{k}^{1}\left(\nu^{k / 2}-1\right)\right] \frac{s^{k}}{k !} \\
a_{k}^{1}=\frac{\pi^{k}}{k} B_{k}, \quad b_{k}^{1}=b_{k}^{0} .
\end{gathered}
$$

Quantities $c_{k}^{i}=a_{k}^{i}\left(1-\theta^{k}\right)+b_{k}^{i}\left(v^{k / 2}-1\right) ; i=0,1$, play the role of semiinvariants and their analogues (for $i=1$ ) with respect to $L_{k}^{i}$. The relation between them is exactly the same as the well-known relation between cumulants and central moments (of course, this remark is nontrivial for $i=1$ ). We have for example:

$$
L_{0}^{i}=1, \quad L_{1}^{i}=0, \quad L_{2}^{i}=c_{2}^{i}, \quad L_{3}^{i}=c_{3}^{i}, \quad L_{4}^{i}=c_{4}^{i}+3\left(c_{2}^{i}\right)^{2} .
$$

THEOREM 3. For any values of $r, k=0,1,2, \ldots$,

$$
(-1)^{r} E \bar{U}^{r} \bar{V}^{k}=L_{k}^{0} \sum^{0}\left(\begin{array}{l}
r \\
j
\end{array}\right) \theta^{r-j}-L_{k}^{1} \sum^{1}\left(\begin{array}{l}
r \\
j
\end{array}\right) \theta^{r-j+1}
$$


where $\Sigma^{0}$ denotes summation over all even and $\sum^{1}$ - summation over all odd values $j$ varying from 0 to $r$.

The proof of the above fact can be reduced to the following. The quantity $\bar{U}^{r}=(U-\theta)^{r}$ is expanded according to the binomial formula, and further we use the fact that $U^{j}=1$ with probability 1 for all even values of $j$.

THEOREM 4. For any integer, positive values of $r, k$ the following equality is true:

$$
\operatorname{cov}\left(\bar{U}^{r}, \bar{V}^{k}\right)=(-1)^{r-1} \sum^{1}\left(\begin{array}{l}
r \\
j
\end{array}\right) \theta^{r-j} \operatorname{cov}\left(\bar{U}, \bar{V}^{k}\right)=(-1)^{r}\left(L_{k}^{o}-L_{k}^{1}\right) \sum^{1}\left(\begin{array}{l}
r \\
j
\end{array}\right) \theta^{r-j+1} .
$$

Proof. We will calculate $E U^{r}$ using the fact that $E U^{j}=1$ for $j$ even and $E U^{j}=0$ for $j$ odd

(3) $E \overline{U^{r}}=\sum_{j=0}^{r}\left(\begin{array}{l}r \\ j\end{array}\right)(-\theta)^{r-j} E U^{j}=(-1)^{r}\left\{\sum^{0}\left(\begin{array}{l}r \\ j\end{array}\right) \theta^{r-j}-\sum^{1}\left(\begin{array}{l}r \\ j\end{array}\right) \theta^{r-j=1}\right\}$.

Using (2), we get

$$
\operatorname{cov}\left(\bar{U}^{r}, \bar{V}^{k}\right)=E \bar{U}^{r} \bar{V}^{k}-E \bar{U}^{r} E \bar{V}^{k}=(-1)^{r}\left(L_{k}^{0}-L_{k}^{1}\right) \sum^{1}\left(\begin{array}{l}
r \\
j
\end{array}\right) \theta^{r-j+1} .
$$

Putting $r=1$, we find that

$$
\operatorname{cov}\left(\bar{U}, \bar{V}^{k}\right)=E \bar{U} \bar{V}^{k}=-\theta\left(L_{k}^{0}-L_{k}^{1}\right) .
$$

The assertion of the theorem easily follows from the above relation. In particular, if $r=4$ it follows from (3) that

$$
E \bar{U}^{4}=1+2 \theta^{2}-3 \theta^{4}
$$

We will now mention the most interesting corollaries which follow from the above facts. We will keep in mind all the time that

$$
B_{2}=\frac{1}{6}, \quad B_{4}=\frac{1}{30} \text { and that } B_{2 k+1}=0 \text { for all } k \geqslant 1 \text {. }
$$

\section{COROLLARY 1.}

The above together with relation (1) gives us the possibility of finding all three parameters describing the distribution of the random variable $X$ :

$$
\nu=\frac{6}{\pi^{2}} D V-\frac{3}{2} D U+1, \quad \theta=E U, \quad \tau=E V .
$$

COROLLARY 2. Random variables $\bar{U}^{r}$ and $\overline{V^{k}}$ are uncorrelated if and only ij one of the following relations holds:

$$
\text { (i) } \theta=0 ; \quad \text { (ii) } L_{k}^{0}=L_{k}^{1} \text {. }
$$

The case where $\theta=0$ is not interesting, as it is connected with an obvious situation. If $\theta \neq 0$, then the above uncorrelation property can only follow from the last condition. Further, as

$$
L_{0}^{i}=1, \quad L_{1}^{i}=0, \quad L_{3}^{0}=L_{3}^{1}
$$

(the last relation holds because $c_{3}^{0}=c_{3}^{1}$ ), then except for the trivial case $k=0$, we get two nontrivial cases for $k=1$ and $k=3$. For any $r$

$$
\left(\bar{U}^{r}, \bar{V}\right) \text { and }\left(\bar{U}^{r}, \bar{V}^{3}\right)
$$

form two pairs of uncorrelated random variables. Condition 2 is not necessarily satisfied for larger $k$. We shall now mention one case where all the above will not be true.

Let $\nu=1$ (it is related to the Cauchy distribution with a linearly transformed argument). Then for all $k$ we have $c_{k}^{i}=a_{k}^{i}\left(1-\theta^{k}\right)$.

Since $a_{k}^{i}=0$ for odd $k$ (because $B_{k}=0$ ), we also have $L_{m}^{i}=0$ for odd $m$. Hence

$$
\operatorname{cov}\left(\bar{U}^{r}, \bar{V}^{k}\right)=0 \quad \text { if } k \text { is odd. }
$$

COROLLARY 3. Recalling the expressions for $D U$ and $D V$ (see (5)), we have

$$
\begin{aligned}
E \bar{U} \bar{V}^{2} & =-\frac{1}{6} \pi^{2} \theta D U, \\
E \bar{V}^{4} & =\frac{1}{8} \pi^{4}\left(1-\theta^{4}\right)+\frac{1}{15} \pi^{4}\left(\gamma^{2}-1\right)+3(D V)^{2}, \\
E \bar{U}^{2} \bar{V}^{2} & =D U D V+\frac{1}{3} \pi^{2} D U(1-D U) .
\end{aligned}
$$

\section{Reduction of the general problem of the parameter estimation}

The general problem of the estimation of the unknown parameters of a stable law $G(x, \alpha, \beta, \gamma, \lambda)$ or a part of those parameters will be broken into two parts.

First part - estimation of the parameters $(\alpha, \beta, \lambda)$ or a part of them, for example estimation of the parameters $(\alpha, \lambda)$.

Second part - estimation of the parameter $\gamma$.

The reason for this division of the general problem is connected with the analytical peculiarities of the stable laws. We can see the essence of those peculiarities when analyzing the properties of the two transformations below.

Let $X_{1}, X_{2}, X_{3}$ be independent random variables having the same stable distribution, described by the parameters $(\alpha, \beta, \gamma, \lambda)$ used in form (A).

Let us generate the following random variables:

$$
X^{0}=X_{1}-X_{2}, \quad X^{*}=X_{1}-\frac{1}{2}\left(X_{2}+X_{3}\right)
$$

One can easily notice that the random variables $X^{0}$ and $X^{*}$ have again stable distributions with parameters univocally defined by the parameters of the distributions of the random variables $X_{j}$.

Namely, we have (see properties (2.3a) and (2.3b)) in form (A):

$$
\begin{gathered}
\alpha^{0}=\alpha, \quad \beta^{0}=0, \quad \gamma^{0}=0, \quad \lambda^{0}=2 \lambda \\
\alpha^{*}=\alpha, \quad \beta^{*}=\left(1-2^{1-\alpha}\right) \beta \lambda / \lambda^{*}, \quad \lambda^{*}=\left(1+2^{1-\alpha}\right) \lambda, \\
\gamma^{*}=0 \quad \text { if } \quad \alpha \neq 1 ; \quad \gamma^{*}=-\lambda \beta \frac{2}{\pi} \log 2 \quad \text { if } \quad \alpha=1 .
\end{gathered}
$$


Note one singularity of such transformation:

$$
\left|\beta^{*}\right| \leqslant\left|1-2^{1-\alpha}\right| /\left(1+2^{1-\alpha}\right) \leqslant \frac{1}{3} .
$$

It is easy to notice that the fact that $X^{0}$ and $X^{*}$ belong to class $\mathfrak{W}$ is the consequence of the above relation.

In order to pass to the system $(\nu, \theta, \tau)$ of the parameters used in the class $\mathfrak{B}$ (form (C)) we should first of all pass to form (B), i.e. act according to the scheme (of mutually univocal relations):

$$
\left(\nu^{*}, \theta^{*}, \tau^{*}\right)_{\mathrm{c}} \leftrightarrow\left(\alpha^{*}, \beta^{*}, \gamma^{*}, \lambda^{*}\right)_{\mathrm{B}}, \quad(\alpha, \beta, \cdot, \lambda) \leftrightarrow\left(\alpha^{*}, \beta^{*}, \gamma^{*}, \lambda^{*}\right)_{\mathrm{A}} .
$$

Parameter $\gamma$ does not appear in those transformations because in passing to the two random variables $X^{0}$ and $X^{*}$ this parameter reduces.

In passing to the random variable $X^{0}$ parameter $\beta$ also reduces.

As a result, in passing to the random variable $X^{0}$ there are only two parameters $(\alpha, \lambda)$ which undergo essential transformations.

$$
(\alpha, \cdot, \cdot \lambda) \leftrightarrow\left(\alpha^{0}, 0,0, \lambda^{0}\right)_{\mathrm{A}}=\left(\alpha^{0}, 0,0, \lambda^{0}\right)_{\mathrm{B}} \leftrightarrow\left(\nu^{0}, 0, \tau^{0}\right)_{\mathrm{C}}
$$

On the other hand, in passing to the random variables $X^{*}$ three parameters $(\alpha, \beta, \lambda)$ undergo essential transformations.

In order to create $n$ independent random variables $X_{1}^{0}, \ldots, X_{n}^{0}$ we should dispose of the samples of the size $2 n$, and to create the same number of random variables $X_{1}^{*}, \ldots, X_{n}^{*}$ - of samples of the size $3 n$ of independent random variables with stable distributions.

After finding the estimates of the parameters $\left(\alpha^{*}, \theta^{*}, \tau^{*}\right)$ (or only $\left(v^{0}, \tau^{0}\right)$ ) we would get the estimates of the parameters $(\alpha, \beta, \lambda)$ in the form interesting for us with the help of the reverse transformations.

Though all those transformations are elementary, in the general case of the three-parameter estimation, however, they are quite complicated. Namely, if the original parameters $(\alpha, \beta, \cdot, \lambda)$ are taken in form (A), and $(\alpha, \beta, \cdot, \lambda)_{\mathrm{A}} \leftrightarrow$ $\left(\alpha^{*}, \beta^{*}, \gamma^{*}, \lambda^{*}\right)_{\mathbf{A}} \leftrightarrow\left(\nu^{*}, \theta^{*}, \tau^{*}\right)_{\mathrm{C}}$ (for the simplicity of the denotations we do not use the star for the estimated parameters, i.e. $\left.\nu=\nu^{*}, \theta=\theta^{*}, \tau=\tau^{*}\right)$, then

$$
\nu=\alpha^{-2}
$$

$$
\begin{aligned}
& \theta=\theta(\alpha, \beta)=\frac{2}{\pi \alpha} \arctan \left[\beta \frac{1-2^{1-\alpha}}{1+2^{1-\alpha}} \tan \left(\frac{\pi}{2} \alpha\right)\right] \quad \text { if } \quad \alpha \neq 1, \\
& \theta(1, \beta)=\lim _{\alpha \rightarrow 1} \theta(\alpha, \beta)=-\frac{2}{\pi} \arctan \left(\beta \frac{\log 2}{\pi}\right) ; \\
& \tau=\tau(\alpha, \beta, \lambda)=\frac{1}{\alpha}\left\{\log \lambda+\frac{1}{2} \log \left[\left(1+2^{1-\alpha}\right)^{2}+\right.\right. \\
& \left.\left.+\beta^{2}\left(1-2^{1-\alpha}\right)^{2} \tan ^{2}\left(\frac{\pi}{2} \alpha\right)\right]+C(1-\alpha)\right\} \quad \text { if } \quad \alpha \neq 1, \\
& \tau(1, \beta, \lambda)=\lim _{\alpha \rightarrow 1} \tau(\alpha, \beta, \lambda)=\log (2 \lambda)+\frac{1}{2}\left[1+\beta^{2}\left(\frac{\log 2}{\pi}\right)^{2}\right] .
\end{aligned}
$$

And conversely

$$
\begin{aligned}
& \begin{array}{l}
\alpha \\
=
\end{array} \frac{1}{\sqrt{\nu}} ; \\
& \beta=\beta(\nu, \theta)=\frac{1+\exp [(1-1 / \sqrt{\nu}) \log 2]}{1-\exp [(1-1 / \sqrt{\nu}) \log 2]} \cdot \frac{\tan \left(\frac{1}{2} \pi \theta / \sqrt{\nu}\right)}{\tan \left(\frac{1}{2} \pi 1 / \sqrt{\nu}\right)} \quad \text { if } \quad \nu \neq 1, \\
& \beta(1, \theta)=\lim _{\nu \rightarrow 1} \beta(\nu, \theta)=-\frac{\pi}{\log 2} \tan \left(\frac{1}{2} \pi \theta\right) ; \\
& \lambda=\lambda(\nu, \theta, \tau) \\
& \quad=\frac{\cos \left(\frac{1}{2} \pi \theta / \sqrt{\nu}\right)}{1+\exp [(1-1 / \sqrt{\nu}) \log 2]} \exp \left[\frac{\tau}{\sqrt{\nu}}-C\left(1-\frac{1}{\sqrt{\nu}}\right)\right] \quad \text { if } \quad \nu \neq 1, \\
& \lambda(1, \theta, \tau)=\lim _{\nu \rightarrow 1} \lambda(\nu, \theta, \tau)=\frac{1}{2} e^{\tau} \cos \left(\frac{1}{2} \pi \theta\right) .
\end{aligned}
$$

However, in passing to the random variables $X^{0}$ (i.e. in estimating only a pair of the parameters $\alpha$ and $\lambda$ ) the original as well as the reversed transformations are not very complicated. Namely, if the original parameters $(\alpha, \cdot, \cdot, \lambda)$ (irrespective of the form) correspond to $\left(\nu^{0}, \theta^{0}, \tau^{0}\right)$, then

$$
\nu^{0}=\alpha^{-2}, \quad \theta^{0}=0, \quad \tau^{0}=\alpha^{-1} \log (2 \lambda)-C\left(1-\alpha^{-1}\right),
$$

where $C$ is the Euler constant. And conversely

$$
\alpha=\left(v^{0}\right)^{-1 / 2}, \quad \lambda=\frac{1}{2} \exp \left\{\left(\tau^{0}+C\right)\left(\nu^{0}\right)^{-1 / 2}-C\right\} .
$$

\section{Estimates of the parameters of stable laws}

In the last section we have seen how the problem of estimation of the parameters $(\alpha, \beta, \lambda)$ of some stable law $G$ can be reduced, in what is in fact an equivalent way, to the problem of parameter estimation of another stable distribution $G^{*}$ belonging to class $\mathfrak{B}$.

We will now be concerned with this problem. That is why we assume at first that $G \in \mathfrak{W}$ and that the parameters $(\alpha, \beta, \lambda)$ correspond in form (C) to the parameters $(\nu, \theta, \tau)$. The well-known method of moments and the relations (1) and (6) are the basis of the estimation of the parameters $(\nu, \theta, \tau)$. Further, we use the following facts well known in statistics (see for example [1]). Let $Y$ be any random variable with a finite fourth moment. Write

$$
a=E Y, \quad b^{2}=D Y, \quad c=E(Y-a)^{4} .
$$

Assume that we are given a sample of $n$ independent observations $Y_{1}, \ldots, Y_{n}$ $(n \geqslant 2)$ of the random variable $Y$.

We create a sample average

$$
A_{Y}=\frac{1}{n} \sum_{1}^{n} Y_{j}
$$


and sample variance

$$
B_{Y}^{2}=\frac{n}{n-1}\left\{A_{Y^{2}}-A_{Y}^{2}\right\} .
$$

Hence $A_{Y}$ and $B_{Y}^{2}$ are the unbiased estimates of $a$ and $b^{2}$, respectively, i.e.

$$
E A_{Y}=a, \quad E B_{Y}^{2}=b^{2} .
$$

Moreover, variances of these estimates can be calculated and have the following form:

$$
\begin{aligned}
& D A_{Y}=\frac{b^{2}}{n}, \\
& D B_{Y}^{2}=\frac{1}{n}\left(c-b^{4}\right)+\frac{2}{n(n-1)} b^{4} .
\end{aligned}
$$

Suppose now that we have at our disposal $n$ independent observations $X_{1}, \ldots, X_{n}$; $n \geqslant 2$ of the random variable $X=X(\nu, \theta, \tau)$ which has a distribution belonging to class $\mathfrak{W}$. We create, using this sample, the following pairs of independent random variables:

$$
\left(U_{j}=\operatorname{sign} X_{j}, V_{j}=\log \left|X_{j}\right|\right) ; \quad j=1,2, \ldots, n .
$$

According to what was presented above, we could take as the estimates of the parameters $(\nu, \theta, \tau)$ the following random variables:

$$
\tilde{\nu}=\frac{6}{\pi^{2}} B_{V}^{2}-\frac{3}{2} B_{U}^{2}+1, \quad \tilde{\theta}=A_{U}, \quad \tilde{\tau}=A_{V} .
$$

The above estimates would have very good properties because $U$ as well as $V$ have finite moments of any order.

This should not be done in general because the region those estimates belong to, i.e.

$$
\tilde{Q}=\left\{(v, \theta, \tau): v \geqslant-\frac{1}{2},|\theta| \leqslant 1,-\infty<\tau<\infty\right\},
$$

turns out to be substantially larger than the region of the admissible values of the estimated parameters (see Fig. 1)

$$
Q=\left\{(\nu, \theta, \tau): \nu \geqslant \frac{1}{4},|\theta| \leqslant \min (1,2 \sqrt{\nu}-1),|\tau|<\infty\right\}
$$

The situation could be improved by such an additional transformation of the random variables $(\tilde{\nu}, \tilde{\theta}, \tilde{\tau})$ into new variables $(\hat{\nu}, \hat{\theta}, \hat{\tau})$ that, on the one hand, range $\hat{Q}$ would be covered by region $Q$, and, on the other hand, that transformation would not damage to much those properties which the random variables $(\tilde{\nu}, \tilde{\theta}, \tilde{\tau})$ had.

This can be done in many ways, and that is why we will choose the additional bounds in such a way as to change as few of the original variables as possible. We should change one of them, however. It turns out that changing only one variable can be sufficient. Namely we change $\tilde{\nu}$ :

$$
\hat{\nu}=\max \left\{\tilde{\nu}, \frac{1}{4}\left(1+|\tilde{\theta}|^{2}\right)\right\}, \quad \hat{\theta}=\tilde{\theta}, \hat{\tau}=\tilde{\tau} .
$$

It can easily be seen that the region of changes of those estimates $\hat{Q}$ is equal to the range $Q$ (the sense of transformation (12) lies in the fact that we transfer the point $\tilde{a}=(\tilde{v}, \tilde{\theta})$ in a parallel manner so that it enters the range $Q)$.

The next step consists in examining the properties of the chosen estimates $(\hat{\nu}, \hat{\theta}, \hat{\tau})$. Write

$$
\sigma_{v}^{2}=D \tilde{\nu}, \quad \sigma_{0}^{2}=D \tilde{\theta}, \quad \sigma_{\tau}^{2}=D \tilde{\tau} .
$$

LEMMA 1. Estimates $\hat{\theta}$ and $\hat{\tau}$ turn out to be unbiased estimates of the parameters $\theta$ and $\tau$ with variances

$$
D \hat{\theta}=\sigma_{0}^{2}=\frac{D U}{n}, \quad D \hat{\tau}=\sigma_{\tau}^{2}=\frac{D V}{n} .
$$

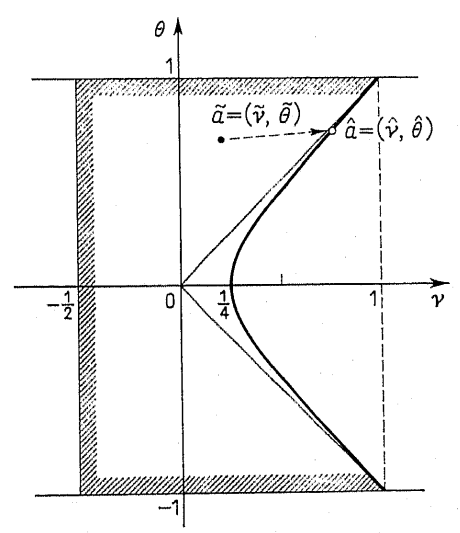

Fig. 1

Besides we have

$$
(E \hat{\nu}-\nu)^{2} \leqslant E(\hat{\nu}-\nu)^{2} \leqslant \sigma_{\nu}^{2}+\sigma_{\theta}^{2} .
$$

The first part of the assertion of the lemma is an easy consequence of the choice of the estimates $\hat{\theta}, \hat{\tau}$ and property (9). Further, since we always have $v=$ $\max \left(\nu, \frac{1}{4}(1+|\theta|)^{2}\right)$

$$
\begin{aligned}
\hat{\nu}-\nu & =\max \left(\tilde{\nu}, \frac{1}{4}(1+|\tilde{\theta}|)^{2}\right)-\max \left(\nu, \frac{1}{4}(1+|\theta|)^{2}\right) \\
& \leqslant \max \left\{\tilde{\nu}-\nu, \frac{1}{4}\left[(1+|\tilde{\theta}|)^{2}-(1+|\tilde{\theta}|)^{2}\right]\right\} \leqslant \max \{\tilde{\nu}-\nu,|\theta-\theta|\} .
\end{aligned}
$$

Moreover, it is obvious that $\hat{\nu}-\nu \geqslant \tilde{\nu}-\nu$. Hence

$$
(\hat{\nu}-\nu)^{2} \leqslant \max \left\{(\tilde{\nu}-\nu)^{2},(\tilde{\theta}-\theta)^{2}\right\} \leqslant(\tilde{\nu}-\nu)^{2}+(\tilde{\theta}-\theta)^{2} .
$$

The second part of the assertion of the lemma can be got from the above.

In order to give the estimates (14) the ultimate form, we should calculate the 
value of the variance $\sigma_{\nu}^{2}$. To do this one needs not only property (10) but also the following general fact concerning sample variances.

We consider any pair of the uncorrelated random variables $(Y, Z)$ which have zero expectations and finite fourth moments. Further, let $\left(Y_{1}, Z_{1}\right), \ldots,\left(Y_{n}, Z_{n}\right)$ be a sample of independent pairs of random variables all distributed as $(Y, Z)$. Let us form the sample variances $B_{Y}^{2}$ and $B_{Z}^{2}$ on the basis of that sample. Then one can prove the following

LeMmA 2. For any $n \geqslant 2$ the following equality holds:

$$
\operatorname{cov}\left(B_{Y}^{2}, B_{Z}^{2}\right)=\frac{1}{n}-\operatorname{cov}\left(Y^{2}, Z^{2}\right) \text {. }
$$

Proof. We will transform the left side of (15) using property (8):

$$
\operatorname{cov}\left(B_{Y}^{2}, B_{Z}^{2}\right)=E B_{Y}^{2} B_{Z}^{2}-D Y D Z \text {. }
$$

We present the first summand in the form of four summands

$$
\begin{aligned}
E B_{Y}^{2} B_{Z}^{2} & =\left(\frac{n}{n-1}\right)^{2} E\left\{A_{Y^{2}} A_{Z^{2}}-A_{Y 2}\left(A_{Z}\right)^{2}-\left(A_{Y}\right)^{2} A_{Z^{2}}+\left(A_{Y}\right)^{2}\left(A_{Z}\right)^{2}\right\} \\
& =\left(\frac{n}{n-1}\right)^{2} E\left\{J_{11}-J_{12}-J_{21}+J_{22}\right\} .
\end{aligned}
$$

We have (the indices $i, j, k, l$ change independently of one another within the bounds from 1 to $n$ ):

$$
\begin{aligned}
& E J_{12}=\frac{1}{n^{3}} \sum E Y_{i}^{2} Z_{j} Z_{k}=\frac{1}{n^{3}} \sum E Y_{l}^{2} Z_{j}^{2}=\frac{1}{n} E J_{11}, \\
& E J_{21}=\frac{1}{n^{3}} \sum E Y_{i} Y_{j} Z_{k}^{2}=\frac{1}{n^{3}} \sum E Y_{i}^{2} Z_{k}^{2}=\frac{1}{n} E J_{11}, \\
& E J_{22}=\frac{1}{n^{4}} \sum E Z_{i} Z_{j} Y_{k} Y_{l}=\frac{1}{n^{4}} \sum Z_{j}^{2} Y_{k}^{2}=\frac{1}{n^{2}} E I_{11} .
\end{aligned}
$$

Hence

$$
\begin{aligned}
E B_{Y}^{2} B_{Z}^{2}=E J_{11} & =\frac{1}{n^{2}} \sum E Y_{i}^{2} Z_{j}^{2}=\frac{1}{n^{2}}\left\{n(n-1) E Y^{2} \cdot E Z^{2}+n E Y^{2} Z^{2}\right\} \\
& =D Y D Z+\frac{1}{n}\left\{E Y^{2} Z^{2}-E Y^{2} E Z^{2}\right\} .
\end{aligned}
$$

The required equality follows, as can easily be seen, from the above relation.

Lemma 3. For all $n \geqslant 2$

$$
\begin{aligned}
\sigma_{p}^{2}=D \tilde{\nu}=\frac{1}{n}\left\{\frac{2}{5}(\nu-1)^{2}+\frac{6}{5}\left(9-5 \theta^{2}\right)(\nu-1)+3\left(1-\theta^{2}\right)\left(3+\theta^{2}\right)\right\}+ \\
+\frac{1}{n(n-1)}\left\{2(\nu-1)^{2}+6\left(1-\theta^{2}\right)(\nu-1)+9\left(1-\theta^{2}\right)^{2}\right\} .
\end{aligned}
$$

Proof. According to (11) we have

$$
\sigma_{\nu}^{2}=D\left(\frac{6}{\pi^{2}} B_{V}^{2}-\frac{3}{2} B_{U}^{2}\right)==\frac{36}{\pi^{4}} D B_{V}^{2}+\frac{9}{4} D B_{U}^{2}-\frac{18}{\pi^{2}} \operatorname{cov}\left(B_{U}^{2}, B_{V}^{2}\right) .
$$

Making use of properties (8) and (9) of the sample variances, we give the following form to the right-hand side of this equality:

$$
\begin{aligned}
\frac{1}{n}\left\{\frac{36}{4}\left[E \bar{V}^{4}-(D V)^{2}\right]\right. & +\frac{9}{4}\left[E \bar{U}^{4}-(D U)^{2}\right]- \\
& \left.-\frac{18}{\pi^{2}} \operatorname{cov}\left(\bar{V}^{2}, \bar{U}^{2}\right)\right\}+\frac{1}{n(n-1)}\left\{\frac{72}{\pi^{4}}(D V)^{2}+\frac{9}{2}(D U)^{2}\right\} .
\end{aligned}
$$

We will transform the first three summands with the help of the expressions of the moments (see (4) and (5))

$$
\frac{36}{\pi^{4}} \cdot\left[E \bar{V}^{4}-(D V)^{2}\right]=\frac{2}{5} W^{2}+6\left(D U+\frac{4}{5}\right) W+9 D U,
$$

where we denote $W=\frac{6}{\pi^{2}} D V-\frac{3}{2} D U$ for the sake of simplicity,

$$
\frac{9}{4}\left[E \bar{U}^{4}-(D U)^{2}\right]=9 D U(1-D U), \quad \frac{18}{\pi^{2}} \operatorname{cov}\left(\bar{U}^{2}, \bar{V}^{2}\right)=6 D U(1-D U) .
$$

As a result we obtain

$$
\begin{aligned}
\sigma_{v}^{2}=\frac{1}{n}\left\{\frac{2}{5} W^{2}+6\left(D U+\frac{4}{5}\right) W+\right. & 3 D U(4-D U)\}+ \\
& +\frac{1}{n(n-1)}\left\{2 W^{2}+6 D U \cdot W+9(D U)^{2}\right\} .
\end{aligned}
$$

It remains to perform the transformation $W=\nu-1, D V=1-\theta^{2}$.

We will now formulate the results of the construction of stable law parameter estimates in the form of the following assertion:

THEOREM 5. The estimates of the parameters $(\nu, \theta, \tau)$ defined according to (11) and (12) on the basis of a sample of size $n$ have the following properties:

1. Estimates $\hat{\theta}$ and $\hat{\tau}$ are unbiased with variances of order $O(1 / n)$ (the exact values for the variances are given in (13) and (15)).

2. Estimate $\hat{v}$ is asymptotically unbiased. Moreover, the square of the bias and the mean square difference between the estimate and the real value of the parameter $v$ have order $O(1 / n)$ (the exact expressions of those number characteristics are given in (13), (14), (16)).

After obtaining estimates $(\nu, \theta, \tau)$, in the transformation chain which brought us to the parameters $(\nu, \theta, \tau)$ (see Section 4) one has to follow the way of the inverse transformations

$$
(\hat{\nu}, \hat{\theta}, \hat{\tau})_{\mathbf{C}} \rightarrow(\hat{\alpha}, \hat{\beta}, \hat{\gamma}, \hat{\lambda})_{\mathbf{B}} \rightarrow\left(\hat{\alpha}, \hat{\beta}, \hat{\gamma}, \hat{\lambda}_{\mathbf{A}} .\right.
$$


Let now $(\alpha, \beta, \cdot, \lambda)_{\mathrm{A}}$ be the parameters of a stable law $G \in \mathfrak{U}$ and $\left(\alpha^{*}, \beta^{*}, \gamma^{*}, \lambda^{*}\right)_{\mathrm{A}}$ the corresponding parameters in the reduced problem, that is,

$$
(\alpha, \beta, \cdot, \lambda)_{\mathrm{A}} \leftrightarrow\left(\alpha^{*}, \beta^{*}, \gamma^{*}, \lambda^{*}\right)_{\mathrm{A}} \leftrightarrow\left(\nu^{*}, \theta^{*}, \tau^{*}\right)_{\mathrm{C}}
$$

In the same way as above we can get an expression for the estimates $\left(\hat{\nu}^{*}, \hat{\theta}^{*}, \hat{\tau}^{*}\right)$ of the parameters $\left(\nu^{*}, \theta^{*}, \tau^{*}\right)$. Unfortunately, in the general case we cannot carry out the inverse transformation of the estimates

$$
\left(\hat{\gamma}^{*}, \hat{\theta}^{*}, \hat{\tau}^{*}\right)_{\mathrm{C}} \rightarrow\left(\hat{\alpha}^{*}, \hat{\beta}^{*}, \hat{\gamma}^{*}, \hat{\lambda}^{*}\right)_{\mathrm{A}}
$$

because the range those estimates belong to, i.e. $\hat{Q}^{*}=\left\{\left(\hat{\nu}^{*}, \hat{\theta}^{*}, \hat{\tau}^{*}\right)\right\}=\hat{Q}$, turns out to be essentially larger than the range of estimated parameters

$$
Q^{*}=\left\{\left(\nu^{*}, \theta^{*}, \tau^{*}\right): \nu^{*} \geqslant \frac{1}{4},\left|\theta^{*}\right| \leqslant H\left(\nu^{*}\right),\left|\tau^{* *}\right|<\infty\right\},
$$

where

$$
H(t)=\frac{2}{\pi} \sqrt{t} \arctan \left\{\frac{\exp [(1-1 / \sqrt{t}) \log 2]-1}{\exp [(1-1 / \sqrt{t}) \log 2]+1} \cdot \tan \left(\frac{\pi}{2} \cdot \frac{1}{\sqrt{t}} \cdot\right)\right\},
$$

if $t \geqslant \frac{1}{4}$ and $t \neq 1$, and

$$
H(1)=\lim _{t \rightarrow 1} H(t)=\frac{2}{\pi} \arctan \left(\frac{\log 2}{\pi}\right) .
$$

$H(t)$ is a continuous and strictly monotone function with $H\left(\frac{1}{4}\right)=0$ and $H(\infty)=\frac{1}{3}$. (see Fig.2),

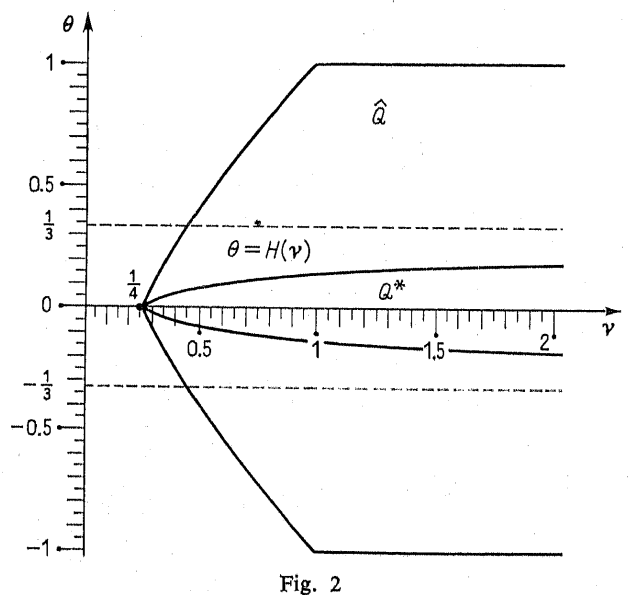

There are some cases where we can use the estimates $(\hat{\nu}, \hat{\theta}, \hat{\tau})$ to construct estimates of the parameters of the original stable law. We shall consider here the simplest two cases. The first is concerned with the construction of the main parameter of the stable law, i.e. $\alpha$. As in the chain of the transformations, we have

$$
\alpha=\alpha_{\mathbf{A}}^{*}=\alpha_{\mathrm{B}}^{*} \leftrightarrow \nu^{*}=\left(\alpha_{\mathrm{B}}^{*}\right)^{-2}=\alpha^{-2} .
$$

Hence the following random variable is the estimate of the parameter $\alpha$ of the original distribution.

$$
\hat{\alpha}=\left(\hat{\nu}^{*}\right)^{-1 / 2}
$$

We encounter of course a similar situation when we decide to pass to the random variables $X^{0}$. The estimate $\hat{\alpha}$ can be constructed in this case with the help of the estimate $\hat{\nu}^{0}$ according to the same formula

$$
\hat{\alpha}=\left(\hat{\nu}^{0}\right)^{-1 / 2} \text {. }
$$

THEOREM 5. Estimate (17), defined on the basis of the sample of size $3 n$, and estimate (18), defined on the basis of the sample of size $2 n$, are asymptotically unbiased estimates of the parameter $\alpha$. The square of the bias and the mean square distance between the estimate and the real value of the parameter is of order $O(1 / n)$. Moreover, the mean square distance between estimate $\hat{\alpha}$ and $\alpha$ is at most four times as great as the mean square distance between $\hat{\nu}$ and $\nu$.

The proof, as can easily be seen, should be made only for the last part of the above theorem. Let $\hat{\alpha}=(\hat{v})^{-1 / 2}$ (where $\hat{\nu}=\hat{\nu}^{*}$ or $\hat{\nu}=\hat{\nu}^{0}$.) As $\alpha=(v)^{-1 / 2}$, we have

$$
|\hat{\alpha}-\alpha|=\frac{|\hat{\nu}-\nu|}{\nu \sqrt{\hat{v}}+\hat{\nu} \sqrt{\nu}} \leqslant 4|\hat{\nu}-\nu|,
$$

because $\nu \geqslant \frac{1}{4}$ and $\hat{\nu} \geqslant \frac{1}{4}$. The last part of the assertion of the theorem obviously follows from the above.

The second case is concerned with estimation of the parameter $\lambda$ when passing to the random variables $X^{0}$. We have here also a very simple chain of transformations of the parameter $\lambda$ :

$$
\lambda \leftrightarrow \lambda_{\AA}^{0}=2 \lambda=\lambda_{\AA}^{\circ} \leftrightarrow \tau^{0} .
$$

One can see from the above that as an estimate of the parameter one can take the following variable:

$$
\hat{\lambda}=\frac{1}{2} \exp \left\{\left(\hat{\tau}^{0}+C\right)\left(\hat{v}^{0}\right)^{-1 / 2}-C\right\} \quad \text { if } \quad \hat{\nu}^{0} \neq 1 .
$$

In the general case we can use, instead of the estimates $\left(\hat{\nu}^{*}, \hat{\theta}^{*}, \hat{\tau}^{*}\right)$ other estimates $\left(\bar{\nu}^{*}, \bar{\theta}^{*}, \bar{\tau}^{*}\right)$ which are constructed with the help of the estimates $\left(\tilde{\nu}^{*}, \tilde{\theta}^{*}, \tilde{\tau}^{*}\right)$ in the following way

$$
\bar{\nu}^{*}=\max \left(\frac{1}{4}, \tilde{\nu}^{*}\right), \quad \bar{\theta}^{*}=\min \left(\tilde{\theta^{*}}, H\left(\bar{\nu}^{*}\right)\right), \quad \bar{\tau}^{*}=\tilde{\tau}^{*} .
$$

It is not difficult to see that the corresponding range of those estimates, $\bar{Q}^{*}$ is equal to the range $Q^{*}$. Hence there is a possibility to carry out the inverse transformation of the estimates $\left(\bar{\nu}^{*}, \theta^{*}, \bar{\tau}^{*}\right)_{C} \rightarrow\left(\bar{\alpha}^{*}, \bar{\beta}^{*}, \bar{\gamma}^{*}, \bar{\lambda}^{*}\right)_{A} \rightarrow(\bar{\alpha}, \bar{\beta}, \cdot, \bar{\lambda})_{A}$.

THEOREM 6. Defined on the basis of the sample of size $3 n$ estimates (19) are asymptotically unbiased estimates of the parameters $\left(\nu^{*}, \theta^{*}, \tau^{*}\right)$ of the corresponding stable law in the reduced problem. The square of the bias and the mean square distance between estimate and real value of each parameter is of order $O(1 / n)$. 
Unfortunately, the analysis of estimates $(\bar{\alpha}, \overline{\beta,} \cdot, \bar{\lambda})$ is an essentially more complicated problem than the analysis of estimates $\left(\bar{\nu}^{*}, \bar{\theta}^{*}, \bar{\tau}^{*}\right)$ and requires a separate consideration. Therefore, the problem of estimation of the parameters of group $(\alpha, \beta, \lambda)$ has a solution which however requires additional analysis.

The problem of estimation of the shift parameter $\gamma$ is based on transformations which are essentially different from those which were used for the estimation of the parameters $(\alpha, \beta, \lambda)$. The point is that there are no known analytical relations in an explicit form with the help of the characteristics of the moments.

Great difficulties involved in the estimation of the parameter $\gamma$ concern also the discontinuity which appears within the set of stable laws at point $\alpha=1$.

A separate paper will be devoted to the problem of obtaining and examining the estimates of the parameter $\gamma$. Also, some experimental results concerning the estimation of the stable law parameters of the group $(\alpha, \beta, \lambda)$ will be given elsewhere.

\section{References}

[1] H. Cra mér, Mathematical methods of statistics, Princeton Univ. Press, Princeton 1946.

[2] E. F. Fa m a and.R. R o 11, Some properties of symmetric stable distributions, J. Amer. Stat. Assoc. 63, N 323 (1968).

[3] -, -, Stable distribution parameters, ibid. 66, N 334 (1971).

[4] B. V. G $\mathrm{nedenko}$ and A. N. Kolmogorov, Limit distributions for sums of independent random variables, Addison-Wesley, Cambridge, Mass. 1954.

[5] И. С. Г р а д шш те й н, И. М. Р ы ж и к, Табличы интегралов, суне, рядов и произведений, Изд. Физ.-мат. лит., Москва 1962.

[6] I.S. Ibramhalilov, On estimates of functionally related parameters, Theory Prob. and Math. Statist. 6 (1975).

[7] W. H. D u M o u $\mathrm{hel}$, On the asymptotic normality of the maximum likelihood estimate when sampling from a stable distribution, Ann. of Statistics 1.5 (1973)

[8] V. M. Z o l o t a rev, Mellin-Stieltjes transformations in probability theory, Theory Probits Appl. 2 (1957).

[9] -, -, On analytical properties of the stable laws, Vestnik Leningradskogo Univ. 1 (1956).

$[10]-,-$, General theory of multiplicity of independent random variables, Doklady Ak. Nauk USSR 142.4 (1962).

[11] - On M-divisibility of stable laws, Theory Prob. its Appl. 12 (1967)

[12] G. J. W o r $\mathrm{s}$ a $1 \mathrm{e}$, The estimation of the symmetric stable distribution parameters, Cowpstat 1976, Proc. Comput. Statistics, 2nd Symposium, Berlin (West), Wien 1976.

[13] A.S. Pa uls o n, E.W. Holcomb, R. A. Leitch, The estimation of the parameters of the stable laws, Biometrika·62.1 (1975).

Presented to the semester

MATHEMATICAL STATISTICS

September 15-December 18, 1976 